\title{
PERCEPÇÃO DE PROFISSIONAIS DE SAÚDE SOBRE O PROGRAMA DE COMBATE À DEFICIÊNCIA DE VITAMINA A
}

\author{
Health professionals' perception of the Brazilian program to \\ combat vitamin A deficiency
}

\author{
Percepción de los profesionales sanitarios sobre el programa \\ de combate a la deficiencia de vitamina $A$
}

Artigo Original

\section{RESUMO}

Objetivo: Identificar a percepção de profissionais que atuam na área de saúde sobre o Programa Nacional de Suplementação de Vitamina A. Métodos: Estudo exploratório, descritivo, de natureza qualitativa, realizado com 15 profissionais de saúde selecionados por amostragem intencional, que trabalhavam em Unidades Básicas de Saúde e maternidades no município de Campina Grande/PB entre os meses de fevereiro e maio de 2010. Para a coleta de dados, utilizou-se a entrevista semidirigida, conduzida por questões norteadoras. $\mathrm{O}$ material foi submetido à Análise de Conteúdo, na modalidade temática. Após análise, emergiram três categorias temáticas. Resultados: A percepção dos entrevistados mostrouse focada na suplementação do micronutriente em detrimento de outras ações pertinentes ao Programa. A falta de capacitação/treinamento, o processo de trabalho fragmentado nas equipes, a irregularidade no fornecimento das cápsulas, a ausência de material educativo e técnico sobre o Programa foram citados como pontos dificultadores e podem contribuir para a não apreensão de sua proposta, como um conjunto de ações articuladas para o combate e/ ou controle da deficiência da vitamina A. Conclusão: Os resultados sinalizam a necessidade de um processo de capacitação crítico e reflexivo para os profissionais de saúde, além da produção e disseminação de material educativo, com vista ao aprimoramento do Programa e sua ampla divulgação, à padronização de normas técnicas e à regularidade na suplementação. Sugere-se que novos estudos sejam realizados envolvendo outros atores do Programa (mães, gestores), com o objetivo de compreender outros fatores que possam contribuir para que o Programa obtenha o impacto esperado.

Descritores: Deficiência de Vitamina A; Pesquisa Qualitativa; Serviços de Saúde.

\section{ABSTRACT}

Objective: To identify the health professionals' perception of the National Vitamin A Supplementation Program. Methods: Exploratory, descriptive and qualitative study, conducted with 15 health professionals selected by intentional sampling, who worked in Primary Health Care Units and maternity units of the city of Campina Grande, Paraiba, Brazil, between the months of February and May 2010. For data collection, a semistructured interview was applied, conducted by guiding questions. Data was submitted to Content Analysis in the thematic mode. After analysis, three thematic categories emerged. Results: The interviewees 'perception was focused on the micronutrient supplementation to the detriment of other relevant actions to the program. The lack of qualification/training, the fragmented work process in the teams, the irregularity of the capsules supply, and the lack of educational and technical material on the program were cited as hindering points, and are liable to contribute to non-understanding of its proposal, as a set of articulated actions for vitamin A deficiency combat and/or control. Conclusion: The results point out the need for a critical and reflexive training process for the health professionals, in addition to the production and spread of educational material aiming at improving the Program and its dissemination, at the technical rules standardization, and the regularity of supplementation. It is suggested that further studies be developed involving other actors within the Program

\author{
Virginia Rossana de Sousa \\ Brito $^{(1)}$ \\ Maria Gorete Lucena de \\ Vasconcelos $^{(2)}$ \\ Alcides da Silva Diniz ${ }^{(2)}$ \\ Inácia Sátiro Xavier França ${ }^{(1)}$ \\ Dixis Figueroa Pedraza ${ }^{(1)}$ \\ Juliane Berenguer de Souza \\ Peixoto $^{(4)}$ \\ Adriana de Azevedo Paiva ${ }^{(3)}$
}

1) Universidade Estadual da Paraíba UEPB - Campina Grande (PB) - Brasil

2) Universidade Federal de Pernambuco UFPE - Recife (PE) - Brasil

3) Universidade Federal do Piauí - UFPI Teresina (PI) - Brasil

4) Secretaria de Saúde do Município de Campina Grande - Campina Grande (PB)

- Brasil

Recebido em: 21/11/2015

Revisado em: 16/01/2016

Aceito em: 17/02/2016 
(mothers, managers), with the aim of understanding other factors that might contribute to the program in the achievement of the expected impacts.

Descriptors: Vitamin A Deficiency; Qualitative Research; Health Services.

\section{RESUMEN}

Objetivo: Identificar la percepción de profesionales que actúan en el área de la salud sobre el Programa Nacional de Suplemento de Vitamina A. Métodos: Estudio exploratorio, descriptivo y de naturaleza cualitativa realizado con 15 profesionales sanitarios elegidos por muestreo intencional y que trabajaban en Unidades Básicas de Salud y maternidades del municipio de Campina Grande/PB entre los meses de febrero y mayo de 2010. Para la recogida de datos se utilizó la entrevista semidirigida que fue conducida por cuestiones norteadoras. Se realizó el Análisis de Contenido en la modalidad temática. Tras el análisis emergieron tres categorías temáticas. Resultados: La percepción de los entrevistados estuvo centrada en el suplemento del micronutriente en vez de otras acciones del Programa. La falta de capacitación/ entrenamiento, el proceso de trabajo dividido en equipos, la irregularidad del suministro de las capsulas, la falta de material educativo y técnico sobre el Programa fueron citados como aspectos que dificultan y que pueden contribuir para la no adquisición de su propuesta como un grupo de acciones articuladas para el combate y/o control de la deficiencia de la vitamina A. Conclusión: Los resultados señalan la necesidad de un proceso de capacitación crítico y reflexivo para los profesionales sanitarios además de la producción y diseminación de material educativo para el perfeccionamiento del Programa y su amplia divulgación, la estandarización de las normas técnicas y la regularidad del suplemento. Sugiérese que se hagan nuevos estudios involucrando otros actores del Programa (madres, gestores) para la comprensión de otros factores que puedan contribuir para el impacto esperado del Programa.

Descriptores: Deficiencia de Vitamina A; Investigación Cualitativa; Servicios de Salud.

\section{INTRODUÇÃO}

A deficiência de vitamina A (DVA) é uma carência nutricional considerada um problema de saúde pública que atinge, principalmente, países economicamente desfavorecidos e está relacionada à pobreza, ao desenvolvimento de processos infecciosos e ao acesso restrito a alimentos fontes de vitamina A. A Organização Mundial de Saúde (OMS) estima que em torno de 19 milhões de gestantes e 190 milhões de crianças apresentem DVA, sendo a África e o sudeste da Ásia as regiões mais afetadas. A DVA prolongada pode levar a desordens no sistema visual, na diferenciação celular, na integridade epitelial, na produção de eritrócitos, na imunidade e na reprodução ${ }^{(1,2)}$.

O Brasil é apontado como uma região de risco à DVA subclínica $^{(3,4)}$. Segundo os resultados da última Pesquisa Nacional de Demografia e Saúde da Criança e da Mulher (PNDS), 17,4\% das crianças brasileiras estavam com DVA em 2006, com maiores taxas no Nordeste (19\%) e Sudeste $(21,6 \%)$ do país ${ }^{(4)}$. Por sua vez, uma revisão de literatura apontou, também, a problemática que a DVA em crianças apresenta para a saúde pública no Brasil ${ }^{(5)}$. Resultado similar foi sistematizado para as crianças brasileiras assistidas em creches $^{(6)}$, e a tendência de manutenção da carência constata-se por dados empíricos mais recentes ${ }^{(7-10)}$.

O Programa Nacional de Suplementação de Vitamina A (PNVITA), instituído em 13 de maio de 2005 por meio da Portaria 729, pelo Ministério da Saúde do Brasil, vem tentando honrar o compromisso assumido pelo país junto às Nações Unidas de controlar a DVA e suas consequências. O Programa destina-se a reduzir e controlar a deficiência em crianças na idade de 6 a 59 meses de idade e puérperas no pós-parto imediato ${ }^{(3)}$. Atualmente, está em fase de expansão por meio do Plano Brasil sem Miséria ${ }^{(11)}$.

A despeito de a DVA se constituir um problema nutricional importante, no cenário nutricional brasileiro, poucos estudos no país se debruçaram sobre a avaliação do processo e dos resultados do Programa ${ }^{(12-14)}$. Na Bahia, uma avaliação centrada em um enfoque multidimensional, alicerçada na tríade estrutura-processo-resultado ${ }^{(12)}$, observou que a maioria das famílias que recebia o benefício relatou não possuir nenhum tipo de conhecimento sobre a vitamina A. Outras lacunas identificadas se referiam à regularidade na distribuição do suplemento, recursos humanos adequadamente treinados e materiais informativos produzidos e distribuídos para a divulgação do Programa.

$\mathrm{Na}$ Paraíba, a literatura apresenta dois estudos ${ }^{(13,14)}$ realizados no âmbito da avaliação do PNVITA. No primeiro estudo $^{(13)}$, o qual considerou a percepção dos responsáveis por crianças menores de cinco anos beneficiadas pelo Programa, observou-se baixo percentual de conhecimento acerca do PNVITA, bem como sobre a vitamina A. No segundo estudo ${ }^{(14)}$, cuja abordagem foi predominantemente quantitativa, identificou-se que a maioria dos serviços não possuía material educativo ou informativo nas UBSF, havia morosidade e insuficiência de acesso às cápsulas de vitamina A em alguns serviços, além da falta de treinamento/capacitação para a maioria dos profissionais de saúde. Pressupõe-se que atores diversos podem interferir nos resultados alcançados pelo PNVITA, e que aspectos que envolvem sua estrutura (treinamento, material técnico, cápsulas) e operacionalização podem ser determinantes para as dificuldades até então assinaladas ${ }^{(14)}$. 
Nesse sentido, considera-se a relevância da DVA como um problema de saúde pública e a importância dos profissionais de saúde no efetivo desempenho do programa de prevenção e controle da DVA.

Diante desse contexto, questiona-se: como os profissionais de saúde apreendem o Programa Nacional de Suplementação de Vitamina A? O presente estudo teve como objetivo identificar a percepção de profissionais que atuam na área de saúde sobre o Programa Nacional de Suplementação de Vitamina A.

\section{MÉTODOS}

Trata-se de um estudo exploratório, descritivo, de natureza qualitativa, realizado em UBSF e maternidades do município de Campina Grande/PB, entre os meses de fevereiro e maio de 2010.

A população elegível foi a de profissionais de saúde (médicos, enfermeiros) ainda técnicos de enfermagem e agentes comunitários de saúde envolvidos com a operacionalização do PNVITA (Programa Nacional de Suplementação de Vitamina A) e com pelo menos um ano de trabalho. A seleção da amostra foi intencional ${ }^{(15)}$, com número de participantes definido pelo critério de saturação teórica ${ }^{(15)}$, que ocorre quando, na coleta de dados, constata-se que elementos novos para subsidiar a teorização, almejada ou possível, não são mais depreendidos a partir do campo de observação da pesquisa. Utilizando esse critério, o estudo foi conduzido por 15 profissionais de oito Unidades de Saúde da Família, escolhidas aleatoriamente e representativas da Estratégia Saúde da Família do município.

O município de Campina Grande localiza-se no agreste paraibano, na parte oriental do Planalto da Borborema. A área urbana corresponde a $87,8 \mathrm{~km}$ de área urbana e 55,6 $\mathrm{km}$ de área rural. Possui uma população, estimada em 2010, de 385.276 habitantes $^{(16) .} \mathrm{O}$ município dispõe de uma rede de serviços de saúde com 58 Unidades Básicas de saúde da Família, uma maternidade pública, um hospital filantrópico e dois hospitais privados com serviços de referência para maternidade, conveniados pelo Sistema Único de Saúde, e uma clínica privada ${ }^{(17)}$.

A coleta dos dados ocorreu por meio de entrevista semidirigida ${ }^{(15)}$, contendo quatro questões norteadoras: Fale-me o que você sabe sobre a vitamina A; O que você conhece sobre a deficiência da vitamina A? O que você percebe sobre o Programa Nacional de Suplementação de Vitamina A? Em sua opinião, quais sãos pontos facilitadores e dificultadores para operacionalizar o Programa?

Para caracterização dos entrevistados, foram coletados dados com as variáveis, sexo, idade, grau de instrução, formação profissional e tempo de atuação no serviço. As entrevistas ocorreram em salas fechadas, cedidas pelas equipes das UBSF e maternidades, com tempo médio de 40 minutos, gravadas em aparelho de áudio.

Utilizou-se a Análise de Conteúdo na modalidade temática ${ }^{(18)}$. Optou-se por elencar as etapas da técnica segundo o autor, o qual as organiza em três fases: 1) préanálise, 2) exploração do material e 3) tratamento dos resultados, inferência e interpretação. A pré-análise é a fase em que se organiza o material a ser analisado, com o objetivo de torná-lo operacional, sistematizando as ideias iniciais. A exploração do material constitui a segunda fase, que consiste na definição de categorias (sistemas de codificação) e na identificação das unidades de registro (unidade de significação a codificar corresponde ao segmento de conteúdo a considerar como unidade base, visando à categorização e à contagem frequencial) e das unidades de contexto nos documentos (unidade de compreensão para codificar a unidade de registro que corresponde ao segmento da mensagem, a fim de compreender a significação exata da unidade de registro). A exploração do material é a fase da descrição analítica, a qual diz respeito ao corpus (qualquer material textual coletado) submetido a um estudo aprofundado, orientado pelas hipóteses e referenciais teóricos. A terceira fase diz respeito ao tratamento dos resultados, inferência e interpretação. Essa etapa é destinada ao tratamento dos resultados. Ocorre nela a condensação e o destaque das informações para análise, culminando nas interpretações inferenciais; é o momento da intuição, da análise reflexiva e crítica ${ }^{(18)}$.

A partir das falas dos entrevistados e com base na Análise do Conteúdo, foi possível apreender as seguintes categorias temáticas: conhecimento limitado sobre a vitamina A e os agravos decorrentes da sua deficiência; (in)visibilidade do Programa; limitações e facilidades operacionais do Programa.

"O projeto de pesquisa foi aprovado pelo Comitê de Ética em Pesquisa da Universidade Estadual da Paraíba, seguindo as determinações da Resolução $n^{0}$. 466/12 do Conselho Nacional de Saúde ${ }^{(19)}$, que regulamenta as pesquisas com seres humanos no Brasil, com CAAE 0050.0.133.000-08. As entrevistas foram realizadas após a assinatura do Termo de Consentimento Livre e Esclarecido, com a garantia do anonimato preservada para os participantes, cognominados com a letra "P" e numeração crescente de 1 a 15."

\section{RESULTADOS E DISCUSSÃO}

Neste espaço serão apresentados os dados relacionados à caracterização dos participantes do estudo e, em seguida, as categorias temáticas que emergiram do estudo. 


\section{Caracterização dos participantes}

Participaram da pesquisa 15 profissionais da área de saúde, 13 atuantes em UBSF e dois em maternidades do município, sendo representados por duas médicas, quatro enfermeiras, três técnicas de enfermagem e seis agentes comunitários de saúde. Os entrevistados foram predominantemente do sexo feminino, com idades de 28 a 52 anos e com a escolaridade que variou entre ensino médio e ensino superior. Com relação ao tempo de atuação do profissional na UBSF/maternidade, a maioria tinha até cinco anos de atividade $(\mathrm{n}=11)$.

As falas e o agrupamento de ideias, analisados de acordo com o objetivo do estudo, levaram à formação de três categorias que representam o contexto da compreensão, percepção e participação de profissionais da área de saúde no PNVITA.

\section{Conhecimento limitado sobre a vitamina A e dos agravos decorrentes da sua deficiência}

Esta categoria, a limitação no conhecimento sobre o micronutriente vitamina A, percebida na fala de alguns profissionais neste estudo, pode interferir nas ações de controle e prevenção da carência nutricional desse micronutriente.

"O que eu sei sobre ela é que é boa para visão... Eu sei que ela fortalece o sistema imunológico, de alguma forma... O que eu sei é isso!” (P8)

"Ela ajuda na visão... E quando a mãe toma, ela passa para o bebê na amamentação...” (P13)

"[...] como eu falei para você, que a vitamina esteja interligada ou a fator hemorrágico ou ao fator de visão da paciente, ou do recém-nascido..." (P12)

Outras funções sobre a vitamina A elencadas apresentaram traços de incerteza que permeiam o conhecimento dos profissionais que atuam no Programa, na aplicação da suplementação e no acompanhamento das doses.

"[...] diminui problemas com a visão da criança, evita cegueira... Ela diminui a diarreia, a infecção respiratória e ajuda no desenvolvimento e no crescimento da criança. Outras coisas, assim que eu sei, por cima, não sei se é certeza, é que é bom para o cabelo, para unhas. Não tenho certeza!" (P5)

Essas constatações assemelham-se às encontradas em estudos anteriores ${ }^{(12,13)}$. Consoante com essa realidade, o Ministério da Saúde tem buscado revisar toda a lógica operacional do Programa, propondo alterações a fim de sensibilizar os profissionais e melhorar a sua divulgação ${ }^{(20)}$. Essas estratégias são importantes, pois, em populações afetadas pela DVA, a suplementação pode provocar uma redução do risco global de morte de $24 \%$ de mortalidade por diarreia, de $28 \%$ de mortalidade por todas as causas e de $45 \%$ em crianças com HIV positivo ${ }^{(3)}$.

O desconhecimento sobre a vitamina A é percebido, também, na fala de profissionais, ao manifestarem preocupação quanto à possibilidade de algumas crianças que recebem a suplementação apresentarem hipervitaminose.

“Já ouvi falar que a vitamina A dá... pressão, aumenta a pressão cerebral e... só. Em crianças saudáveis... não tem muita necessidade, não.” (P10)

"Você pode ter danos cerebrais. Em alto nível, ela traz danos também." (P9)

Esclarecimentos nesse sentido são extremamente necessários. A suplementação com vitamina $\mathrm{A}$ em áreas em que a DVA constitui um problema de saúde pública é uma estratégia vital para o aumento da sobrevivência de crianças menores de cinco anos ${ }^{(1-3)}$. Vale destacar que a suplementação dessa vitamina pode produzir sintomas como abaulamento da fontanela, cefaleia, tontura e vômito, mas isso se apresenta em percentual muito pequeno ${ }^{(1)}$ quando os critérios preconizados como faixa etária, dose, intervalo e grupos ${ }^{(1,3)}$ são respeitados.

Efeitos outros, como teratogênicos, são atribuídos ao ácido retinoico, e não ao retinol, forma administrada na suplementação. Além da recomendação de ser administrada, especificamente, com a mulher ainda na maternidade ${ }^{(1,3)}$.

O PNVITA possui várias ações para a prevenção e o controle da DVA, sendo a administração do suplemento apenas uma ação em curto prazo. O conhecimento sobre a vitamina A é importante para que o profissional que trabalha com o Programa possa reconhecer os benefícios da suplementação e contribuir com uma prática crítica e coerente com o impacto esperado ${ }^{(1,3)}$.

\section{(In)visibilidade do Programa de Vitamina A}

Esta categoria trata da tênue visibilidade em relação ao PNVITA e seu público-alvo, perceptível na fala dos profissionais, quando questionados sobre o conhecimento do Programa.

"Na verdade, eu nem sabia que existia esse Programa da Vitamina A, [nem sabia] que tinha tanta importância assim, não." (P15)

"[...] Para ser feita em crianças a partir de seis meses... Até um ano, né? Até dois anos? Até dois anos!" (P2)

“[...] Agora, assim, não é a gestante, é a puérpera que é feita, que está sendo feita em seguida ao parto...” (P7)

Esses resultados corroboram com os obtidos em outros trabalhos ${ }^{(12,13)}$. A situação encontrada é crítica não 
apenas na conjuntura específica relacionada à importância da capacitação, mas também em relação à necessidade de acompanhar as mudanças na execução do Programa, como a inclusão das puérperas no grupo da população alvo e o registro de Saúde da Criança. A capacitação sobre o PNVITA é essencial para garantir a cobertura e operacionalização adequadas à proposta do Programa. É importante que profissionais conheçam e usem os materiais operacionais e outros produzidos pela Coordenação Geral de alimentação e Nutrição do Ministério da Saúde, disponíveis no site desse segmento $^{(3,13,14)}$.

\section{Limitações e facilidades operacionais do Programa}

Esta categoria evidencia algumas limitações e facilidades pontuadas para o desenvolvimento das atividades do Programa.

Entre as limitações, está a fragmentação do processo de trabalho revelada nas seguintes falas:

"A gente só acompanha as de tétano, então, as de vitamina $A$, a gente não sabe como é que é, como é que está isso. Quem sabe, no caso, é a enfermeira e a auxiliar." (P1)

“[...] Não tem nada que a gente tenha sentado, que tenha inclusive se reunido com a técnica... [...]. A não ser que tenha feito com outros profissionais da equipe, mas com os técnicos [...]. São os técnicos que, [n] a maioria das vezes, manuseia[m] a vitamina $A$ e faz [em] a vitamina, quase em 100\% das Unidades..." (P6)

Percebe-se, nas falas, que o processo de trabalho dos profissionais mantém uma lógica taylorista de divisão e organização das atividades, como identificado por outros pesquisadores $^{(20)}$. Essas circunstâncias prejudicam a gestão e o processo de trabalho em saúde, bem como a eficácia da Estratégia Saúde da Família, que supõe o trabalho em equipe e a interação organizada entre os profissionais. Produz-se, assim, um desgaste intenso e a precarização do trabalho em saúde, que vem sendo apontado como um importante obstáculo para o desenvolvimento dos serviços públicos no Brasil( ${ }^{(21,22)}$.

Esse cenário requer do profissional posicionamento crítico e reflexivo, necessário para que as mudanças possam surgir a partir das lacunas identificadas nesse processo. A atuação crítico-reflexiva dos profissionais de saúde pode contribuir para a elaboração de estratégias necessárias à prática qualificada em saúde. Nesse sentido, a educação permanente constitui uma ferramenta importante não só para a aquisição e aprimoramento de conhecimentos, mas também para o desenvolvimento de postura crítica, autoavaliação, autoformação e autogestão(23).

Entre os fatores debatidos, as condições de trabalho foram colocadas como importantes limitações relacionadas ao desenvolvimento das ações.
"[...] Que a gente recebesse pelo menos informações, já que, se não é possível o treinamento, pelo menos recebesse um manual, algo que a gente tivesse para tirar uma dúvida." (P10)

"Às vezes existe a falta, principalmente da vitamina A de 100, sempre tem falta na Unidade, mas a gente sempre está procurando fazer." (P6)

Essa percepção reflete a realidade dos serviços públicos de saúde no país, caracterizados por uma estagnação relacionada à reestruturação da rede de serviços e pela inadequação em relação aos materiais e insumos de trabalho ${ }^{(24)}$. Essa situação é preocupante, pois a adequação da estrutura é indispensável para um bom processo de trabalho, com efeitos positivos na gestão e nas condições de trabalho ${ }^{(20,24)}$.

Outro fator percebido como limitador do desenvolvimento do Programa foi a falta de capacitação/ treinamento, como descrito a seguir:

"Para ser sincero, a gente administra, mas a gente não tem informação nenhuma. Fica até ruim para a gente repassar para as pessoas que procuram saber, até para as próprias mãezinhas, entendeu?" (P12)

"Eu tenho medo desses programas que vêm de cima pra baixo. E a gente faz sem ter o conhecimento, porque eu acho que quando você terminar esse negócio, você vai ver que nem as enfermeiras $e$ as auxiliares de enfermagem sabem por que fazem a vitamina A. E só fazem cobrar (a gestão), porque é cobrado pra gente! É cobrado vitamina A pra gente porque senão fica com o calendário de vacina atrasado. Por quê? O beneficio? O que pode causar?" (P9)

Por outro lado, quando os profissionais chegam a participar de alguma atividade educativa, o uso da metodologia desenvolvida é outra questão observada:

"Os cursos que a gente faz é tudo curso virtual, certo? Se você quiser, você tem que ter o seu pen-drive para copiar as aulas, que não são aulas direcionadas para a construção do conhecimento, é aquela metodologia tradicional da universidade capenga." (P4)

Esses resultados convergem com os de revisões recentes que destacam a falta de capacitação como barreira para o desenvolvimento de ações no contexto da Estratégia de Saúde da Família, como a vigilância do crescimento $^{(25)}$ e o preenchimento da Caderneta de Saúde da Criança ${ }^{(26)}$. Nesse sentido, a Política Nacional de Educação Permanente em Saúde se constitui um recurso indispensável à transformação das concepções e práticas dos trabalhadores dos serviços de saúde, colocando-os como atores reflexivos, construtores de conhecimento e de alternativas de ação ${ }^{(27)}$. Não obstante, o que se observa na implementação de políticas ou programas 
de saúde são capacitações ou treinamentos com ações prescritivas, que priorizam o conhecimento especializado e desconsideram as capacidades, limites e possibilidades locais ${ }^{(28)}$. Essa realidade está representada também no contexto do PNVITA, com a execução de ações pontuais, em vez de estarem inseridas em uma política pública de promoção da saúde, com prejuízos em práticas importantes como a educação nutricional ${ }^{(29)}$.

Instigados para discorrer sobre os pontos facilitadores do Programa, os profissionais não teceram muitas considerações, apenas que a administração oral das cápsulas é de fácil aceitação pela população. Ao serem indagados(as) sobre quais sugestões poderiam citar para o aprimoramento do Programa, foram unânimes em considerar a necessidade de capacitação/treinamento e divulgação, o que converge com os resultados antepostos e discutidos.

"[...] Capacitação para os profissionais de saúde e, depois, haver mais divulgação... da importância dessa vitamina, porque eu acho que não tem." (P3)

"Eu nunca fiz um estudo sobre a vitamina A, não, até porque, quando a gente entra, eles nunca incluem muito a vitamina A para fazer acompanhamento." (P11)

A ausência de treinamento dos profissionais de saúde, identificada nas falas, pode repercutir negativamente na operacionalização das ações necessárias do Programa. Questão que deve provocar, nos profissionais e na gestão da Saúde, a necessidade de garantir a ampliação do conhecimento sobre a problemática da DVA e das ações que devem ser pactuadas pelos municípios, estados e Ministério da Saúde ${ }^{(1,2,3,5)}$

\section{CONSIDERAÇÕES FINAIS}

O estudo mostra que a apropriação do conhecimento técnico sobre a vitamina $\mathrm{A}$ e os efeitos deletérios da sua deficiência são limitados. A percepção desses profissionais de saúde sobre o PNVITA indicou a supremacia da administração das doses do suplemento da vitamina A como uma ação isolada.

A falta de capacitação/treinamento, o processo de trabalho fragmentado nas equipes, a irregularidade no fornecimento das cápsulas, a ausência de material educativo e técnico, citados como pontos dificultadores, podem estar contribuindo para a não apreensão da proposta do Programa como um conjunto de ações articuladas para o controle da DVA.

Portanto, os resultados deste estudo apontam a necessidade de treinamento/capacitação dos profissionais de saúde. Ressalta-se a necessidade de a capacitação remeter a um exercício conjunto que legitime os direitos dos seus beneficiários e participação dos mesmos no Programa.
Sugere-se que novos estudos sejam realizados envolvendo outros atores do Programa, com o objetivo de compreender, a partir dos seus espaços de interseção, outros fatores que podem obstaculizar o seu impacto.

\section{AGRADECIMENTOS}

Aos profissionais que participaram do estudo e à Fundação de Apoio e Pesquisa do Estado da Paraíba/ FAPESQ-PB.

\section{REFERÊNCIAS}

1. Organização Mundial da Saúde - OMS. Diretriz: suplementação de vitamina A em bebês e crianças de 6-59 meses de vida. Genebra: Organização Mundial da Saúde; 2013.

2. World Health Organization. WHO technical consultation on vitamin A in newborn health: mechanistic studies. Geneva: WHO; 2012.

3. Ministério da Saúde (BR). Manual de Condutas gerais do Programa Nacional de Suplementação de Vitamina A [Internet]. Brasília: Ministério da Saúde; 2013 [acesso em 2013 Set 10]. Disponível em: nutrição. saude.gov.br/vita.php

4. Ministério da Saúde (BR). Pesquisa Nacional de Demografia e Saúde da Criança e da Mulher - PNDS 2006: resultados sobre anemia e hipovitaminose no Brasil. Brasília: Ministério da Saúde; 2009.

5. Ramalho A, Padilha P, Saunders C. Análise crítica de estudos brasileiros sobre deficiência de vitamina A no grupo materno-infantil. Rev Paul Pediatr 2008:26(4):392-9

6. Pedraza DF, Rocha ACD. Deficiências de micronutrientes em crianças brasileiras assistidas em creches: revisão da literatura. Ciênc Saúde Coletiva. 2016;21(5):1525-43.

7. Silva LLS, Peixoto MRG, Hadler MCCM, Silva AS, Cobayashi F, Cardoso MA. Estado nutricional de vitamina $\mathrm{A}$ e fatores associados em lactentes atendidos em Unidades Básicas de Saúde de Goiânia, Goiás, Brasil. Rev Bras Epidemiol. 2015;18(2):490-502.

8. Gondim SSR, Diniz AS, Cagliari MPP, Araújo ES, Queiroz D, Paiva AA. Relação entre níveis de hemoglobina, concentração de retinol sérico e estado nutricional em crianças de 6 a 59 meses do Estado da Paraíba. Rev Nutr. 2012;25(4):441-9.

9. Azevedo MMS, Cabral PC, Diniz, AS, Fisberg M, Fisberg RM, Arruda IKG. Deficiência de vitamina A 
em pré-escolares da cidade do Recife, Nordeste do Brasil. Arch Latinoam Nutr. 2010;60(1):36-41.

10. Figueroa Pedraza D, Queiroz D, Paiva AA, Cunha MAL, Lima ZN. Seguridad alimentaria, crecimiento y niveles de vitamina $A$, hemoglobina y zinc en niños preescolares del nordeste de Brasil. Ciênc Saúde Coletiva. 2014;19(2):641-50.

11. Ministério da Saúde (BR). Plano Brasil sem miséria no seu município. Brasília: Ministério da Saúde; 2013 [acesso em 2015 Ago 21]. Disponível em: http:// aplicacoes.mds.gov.br/sagi/RIv3/dadosSv/cartilha $\mathrm{msm} /$ cartilha.pdf

12. Martins MC, Santos LMP, Santos SMC, Araújo MPN, Lima AMP, Santana, LAA. Avaliação de políticas de segurança alimentar e combate à fome no período 1995-2002.3 - O Programa Nacional de Controle de Vitamina A. Cad Saúde Pública. 2007;23(9): 2081-93.

13. Almeida ER, Carvalho AT, Nilson EAF, Coutinho, JG, Ubarana JA. Avaliação participativa do Programa Nacional de Suplementação de Vitamina A em um município da Região Nordeste do Brasil. Cad Saúde Pública. 2010;26(5):949-60.

14. Paiva AA, Cagliari MPP, Queiroz D, Souto RA, Brito, VRS, França ISX. Programa Nacional de Suplementação de Vitamina A no Estado da Paraíba: uma análise a partir do relato de profissionais da Equipe de Saúde da Família. Epidemiol Serv Saúde. 2011;20(3):373-83.

15. Fontanella BJB, Luchesi BM, Saidel MGB, Ricas, J, Turato ER, Melo DG. Amostragem em pesquisas qualitativas: proposta de procedimentos para constatar saturação teórica. Cad Saúde Pública. 2011;27(2):38994.

16. Instituto Brasileiro de Geografia e Estatística - IBGE. Censo 2010. Cidades V [acesso em 2011 Jan 22]. Disponível em: http://www.ibge.gov.br/cidadesat/ topwindow.htm?1

17. DATASUS. Cadastro Nacional de Estabelecimentos de Saúde [acesso em 2011 Jan 22] Disponível em: http:// cnes. datasus.gov.br/od-nd-equipes.asp?VEstado $=25 \&$ VMun $=2504008 \&$ VComp $=$

18. Bardin L. Análise de Conteúdo. Lisboa: Edições 70; 2011.

19. Ministério da Saúde (BR), Conselho Nacional de Saúde (CNS), Comissão Nacional de Ética em Pesquisa. Resolução $n^{\circ}$ 466/2012. Normas regulamentadoras de pesquisa envolvendo seres humanos. Brasília: CNS; 2012.
20. Shimizu HE, Carvalho DA Junior. O processo de trabalho da Estratégia Saúde da Família e suas repercussões no processo saúde-doença. Ciênc Saúde Coletiva. 2012;17(9):2405-14.

21. Chiavegato LG Filho, Navarro VL. A organizacão do trabalho em saúde em um contexto de precarização e do avanço da ideologia gerencialista. Rev Pegada. 2012;13(2):67-82.

22. Lacerda MR, Gomes IM, Kalinowski LC, Giacomozzi CM. Estratégias para avanços na prática do cuidado domiciliar. Cogitare Enferm. 2010;15(4):609-15.

23. Silva LA, Casotti CA, Chaves SCL. A produção científica brasileira sobre a Estratégia Saúde da Família e a mudança no modelo de atenção. Ciênc Saúde Coletiva. 2013;18(1):221-32.

24. Laporte-Pinfild ASC, Zangirolani LTO, Spina N, Martins PA, Medeiros MAT. Atenção nutricional no pré-natal e no puerpério: percepção dos gestores da Atenção Básica à Saúde. Rev Nutr. 2016;29(1):109-23 .

25. Figueroa PD. Growth surveillance in the context of the primary public healthcare service network in Brazil: literature review. Rev Bras Saúde Matern Infant. 2016;16(1):7-19.

26. Abreu TGT, Viana LS, Cunha CLF. Desafios na utilização da caderneta de saúde da criança: entre o real e o ideal. J Manag Prim Health Care. 2012;3(2):80-3.

27. Ministério da Saúde (BR). Política nacional de educação permanente em saúde. Brasília: Ministério da Saúde; 2009.

28. Miccas FL, Batista SHSS. Educação permanente em saúde: metassíntese. Rev Saúde Pública. 2014;48(1):170-85.

29. Rodrigues LPF, Roncada MJ. A educação nutricional nos programas oficiais de prevenção da deficiência da vitamina A no Brasil. Rev Nutr. 2010;23(2):297-305.

\section{Endereço para correspondência:}

Virginia Rossana de Sousa Brito

Universidade Estadual da Paraíba - UEPB

Programa de Pós-Graduação em Saúde Pública

Avenida das Baraúnas, 351

Bairro: Campus Universitário, Bodocongó

CEP: 58.101.001. Campina Grande (PB), Brasil

E-mail:vrossana@uol.com.br 